\title{
Our panel of experts highlight the most important research articles across the spectrum of topics relevant to the field of CNS oncology
}

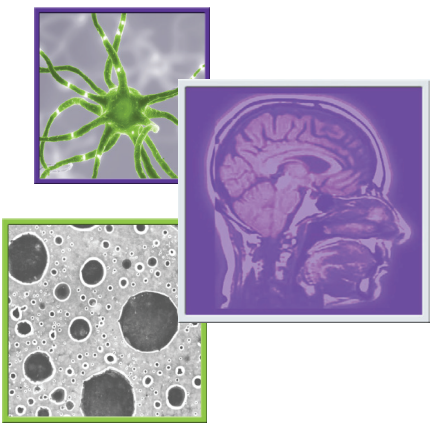

Expert panel: Larry Junck, University of Michigan Health System, MI, USA; Maura Massimino, Fondazione Istituto di Ricovero \& Cura a Carattere Scientifico Istituto Nazionale dei Tumori, Milan, Italy

Possible new therapy for DIPG

- Hashizume R, Andor N, Ihara Y, Lerner R, Gan $\mathrm{H}$, Chen $\mathrm{X}$ et al. Pharmcologic inhibition of histone demethylation as a therapy for pediatric brainstem glioma. Nat. Med. 20(12), 1394-1396 (2014).

Diffuse intrinsic pontine glioma, DIPG, are genetically distinguished in the majority of cases by an oncogenic mutation of the H3F3A gene, which encodes histone variant H3.3. This is considered the driving mutation that reduces histone K27 methylation and thereby alters gene expression in cells of the developing pons. The hypothesis behind the experience described in this paper was that pharmacologic reversal of brainstem glioma H3K27 demethylation could serve as a therapeutic strategy in this disease that is generally lethal within 1 year. The therapy was not targeting the mutant protein directly but rather inhibiting a wild-type enzyme of the methylation pathway, hence promoting the methylation of the nonmutant protein allele. Treatment of all cell sources used in these experiments with GSKJ4, an ethyl ester derivative of the H3K27 demethylase inhibitor GSKJ1 revealed a dosedependent inhibition of cellular viability and completely inhibited the clonal growth of all K27M-expressing cells. The experiment was repeated in vivo, in mice harboring subcutaneous DIPG xenografts, demonstrating significant growth-inhibition activity. The drug was also appreciated to cross the blood-brain barrier.

This is the first report of a target therapy specifically acting on the recently discovered [1] specific DIPG mutation.
Long-term survivors with low-grade gliomas

- Nwachukwu CR, Youland RS, Chioreso C, Wetjen N, Nageswara Rao A et al. Health related quality of life (HRQOL) in longterm survivors of pediatric low grade gliomas (LGGs). J. Neurooncol. 121(3), 599-607 (2015)

This paper reports the health-related quality of life - HRQOL - in a very large cohort of longterm survivors of pediatric low-grade gliomas. Median follow-up was very long, over 20 years. The authors, through patient responses to specific QOL questionnaires, analyzed a series of parameters and their relation to final outcome. In multivariable analysis, radiation therapy was the only independent predictor for physical and role functioning as well as symptoms related to brain tumors such as visual disorders and motor dysfunction. In comparison with previously reported series on adults with LGG, this cohort had overall better QOL scores maybe because, as the authors comment, when time since treatment end is longer, long-term survivors tend to document a better quality of life. Even if radiation therapy was associated with lower physical and role functioning, there was no association with lower self-reported cognitive function in this study. The scores of this whole population were very similar to those of a reference population of healthy adults. These data are of great interest because they derive from a big patient population. The future studies will have to include also the meaning of rehabilitation interventions in patients' stories.

- Written by Maura Massimino
Future : Medicine $\%$ 
Anorexia and fourth ventricle astrocytoma

- Leroy HA, Baroncini M, Delestret I, Florent

$V$, Vinchon M. Anorexia: an early sign of fourth ventricle astrocytoma in children. Childs Nerv. Syst. 30, 2089-2095 (2014).

The authors describe anorexia as a rather significant symptom in the clinical presentation of posterior fossa astrocytoma. In a 12-year retrospective series, 7/34 low-grade glioma patients presented with a story of anorexia associated with an average BMI below the second SD and a prediagnostic duration of almost 1 year. These prodromic signs are obviously to be distinguished from Russel syndrome, the 'failure to thrive' that is the characteristic feature of centrally located diencephalic low-grade gliomas. This poorly documented story of fourth ventricle tumor anorexia is hypothesized to be dependent on leptin secreted by tumor. Leptin is an anorexigenic adipocyte-derived hormone discovered in 1994 that, if secreted by the tumor, could justify the prediagnostic anorexic period followed by the appetite rescue after surgery. The final demonstration of this hypothesis could be given only by leptin bioassay before and after tumor resection.

- Written by Maura Massimino

Whole-brain radiotherapy with

hippocampal avoidance

- Gondi V, Pugh SL, Tome WA et al.

Preservation of memory with conformal avoidance of the hippocampal neural stem-cell compartment during wholebrain radiotherapy for brain metastases (RTOG 0933): a Phase II multi-institutional trial. J. Clin. Oncol. 32, 3810-3816 (2014).

This is a single-arm Phase II trial, RTOG 0933, of whole brain radiotherapy (WBRT) for brain metastases using hippocampal avoidance (HA) to minimize injury to hippocampal neural stem cells. Patients were required to have brain metastases outside a $5 \mathrm{~mm}$ margin around the hippocampi. Radiotherapy was delivered using intensity modulated radiotherapy to $30 \mathrm{~Gy}$ in ten fractions, planned so that the dose to $100 \%$ of the hippocampus did not exceed 9 Gy. For the 42 patients analyzable at 4 months by the Hopkins Verbal Learning Test-Revised, Delayed Recall, the primary end point, average score declined by $7 \%$ at 4 months compared with $30 \%$ decline in a historical control study of standard WBRT from 2003 ( $\mathrm{p}<0.001)$. Three patients had progression in the HA area. Median survival was 6.8 months compared with 4.9 months in the control study. This single-arm study suggests that WBRT with HA may have less effect on memory than standard WBRT, without significantly compromising efficacy. Two randomized studies are planned to further evaluate this possibility.

- Written by Larry Junck

The BELOB Phase 2 trial for recurrent glioblastoma

- Taal W, Oosterkamp HM, Walenkamp

AME et al. Single-agent bevacizumab

or lomustine versus a combination of

bevacizumab plus lomustine in patients

with recurrent glioblastoma (BELOB trial):

a randomized controlled Phase 2 trial. Lancet

Oncol. 15, 943-953 (2014).

This article reports the results of a randomized Phase II study for first recurrence of glioblastoma in which 153 patients were assigned to receive bevacizumab $10 \mathrm{mg} / \mathrm{kg}$ every 2 weeks, lomustine $110 \mathrm{mg} / \mathrm{m}^{2}$ every 6 weeks or bevacizumab $10 \mathrm{mg} / \mathrm{kg}$ every 2 weeks plus lomustine $110 \mathrm{mg} /$ $\mathrm{m}^{2}$ every 6 weeks (later reduced to $90 \mathrm{mg} / \mathrm{m}^{2}$ for safety reasons). Survival at 9 months, the primary outcome measure, was $43 \%$ (95\% CI: 29-57) in the lomustine group, 38\% (95\% CI: 25-51) in the bevacizumab group, 63\% (95\% CI: 49-75) for the combined bevacizumab and lomustine groups (both dose levels). The authors conclude that the combination of bevacizumab and lomustine shows promise for recurrent glioblastoma. Toxicity was generally acceptable. The lomustine and lomustine plus bevacizumab arms will continue enrolling patients in European Organisation for Research and Treatment of Cancer study 26101, an adequately powered randomized Phase III trial, to confirm and extend these findings.

- Written by Larry Junck

Financial \& competing interests disclosure The authors have no relevant affiliations or financial involvement with any organization or entity with a financial interest in or financial conflict with the subject matter or materials discussed in the manuscript. This includes employment, consultancies, honoraria, stock ownership or options, expert testimony, grants or patents received or pending, or royalties.

No writing assistance was utilized in the production of this manuscript. 
JOURNAL WATCH

Reference

1 Schwartzentruber J, Korshunov A, Liu XY et al. Driver mutations in histone $\mathrm{H} 3.3$ and chromatin remodelling genes in paediatric glioblastoma. Nature 482, 226-231 (2012). 